\title{
Dolicomega da artéria vértebro-basilar como causa de perda auditiva neurossensorial assimétrica - relato de caso
}

\section{Megadolicho basilar artery as a cause of asymmetrical sensorineural hearing loss - case report}

\author{
Antonio Antunes Melo', Fernando Souza Leão², Alexandre José Costa Campos², Maria do Rosário Távora de Arruda Antunes ${ }^{3}$, \\ Débora Bunzen², Sílvio da Silva Caldas Neto ${ }^{4}$.
}

1) Doutorado. Otorrinolaringologista do Serviço de Otorrinolaringologia da Universidade Federal de Pernambuco.

2) Especialista em Otorrinolaringologia. Otorrinolaringologista.

3) Especialista em Fonoaudiologia.Fonoaudióloga.

4) Livre Docencia. Professor Adjunto de Otorrinolaringologia da Universidade Federal de Pernambuco.

Instituição: Serviço de Otorrinolaringologia do Hospital das Clínicas da Universidade Federal de Pernambuco. Recife / PE - Brasil

Endereço para correspondência: Antonio Antunes Melo - Rua Dom João de Souza 40/2102 - Madalena - Recife / PE - Brasil - CEP: $50610-070$ - Telefax: (+55 81) 3492-2695-E-mail: antunes.orl@gmail.com

Artigo recebido em 17 de Julho de 2009. Artigo aprovado em 28 de Abril de 2011

\section{RESUMO}

Introdução: No diagnóstico diferencial das perdas sensorioneurais assimétricas fazem parte os distúrbios vasculares e dentre essas alterações encontra-se o dolicomega da artéria vértebro-basilar. Habitualmente essa doença é assintomática e quando há sintomas esses podem ser causados por compressão ou isquemia. Clinicamente podem ocorrer: perda neurossensorial, zumbido, cefaleia, hipoestesia facial, neuralgia trigeminal, vertigem, diplopia e paralisia facial entre outros. O exame de imagem de escolha para seu diagnóstico é a ressonância nuclear magnética. A terapia do dolicomega da artéria basilar pode ser intervencionista ou conservadora dependendo dos achados associados. A abordagem multidisciplinar incluindo neurologista, neurocirurgião e otorrinolaringologista para adequada condução do caso é recomendada.

Objetivo: Relatar o caso de um paciente com perda auditiva neurossensorial assimétrica cujo diagnóstico foi de dolicomega da artéria basilar.

Relato do Caso: JBS, 57 anos, sexo masculino, branco com história de hipoacusia neurossensorial assimétrica e zumbido tipo apito bilateral há vários anos. Exame otorrinolaringológico apresentando otoscopia, rinoscopia anterior e orofaringe normais.

Comentários Finais: $\mathrm{O}$ tratamento consistiu de acompanhamento do paciente, controle do zumbido com medicação e uso de um aparelho de amplificação sonora individual na orelha esquerda.

Palavras-chave: artéria basilar, zumbido, perda auditiva neurossensorial.

\section{SUMMARY}

Introduction: At the differentiated diagnosis of asymmetrical sensorineural hearing losses, vascular disorders are present, one of which is megadolicho basilar artery. This disease is generally asymptomatic, and when symptoms are found, they can be caused by a compression or ischemia. Clinically, sensorineural hearing loss, tinnitus, headache, facial hypoesthesia, trigeminal neuralgia, vertigo, diplopia and facial palsy, among others, are likely to occur. The image examination of choice for its diagnosis is nuclear magnetic resonance. The megadolicho basilar artery therapy can be surgical or conservative, according to the associated findings. A multidisciplinary approach, including a neurologist, neurosurgeon and an otorhinolaryngologist is recommended for a proper administration of the case.

Objective: Report the case of a patient with asymmetrical sensorineural hearing loss, diagnosed of megadolicho basilar artery.

Case report: JBS, 57-year-old white male with a history of asymmetrical sensorineural hearing loss and bilateral whistlelike tinnitus for several years. The otorhinolaryngologic evaluation, including otoscopy, anterior rhinoscopy and oral pharynx, was normal.

Final Comments: The treatment consisted in following up with the patient, controlling the tinnitus by drugs and using an individual sound amplification apparatus on the left ear. Keywords: basilar artery, tinnitus, sensorineural hearing loss. 


\section{INTRODUÇÃO}

Oachado de uma perda neurossensorial assimétrica deve sempre levantar um conjunto de hipóteses diagnósticas, bem como a elaboração de um raciocínio clínico direcionado para investigar as diversas possíveis causas. As doenças retrococleares fazem parte do diagnóstico diferencial das perdas auditivas neurossensoriais, tais como: neurinomas do acústico, meningiomas, cistos epidermoides intradurais congênitos, schwannomas nãoacústicos da fossa posterior, dolicoectasias vértebro-basilares, aneurismas, malformações artério-venosas, lipomas, hemangiomas e osteomas $(1,2)$.

Na investigação desses casos podem ser solicitados para avaliação os seguintes exames: estudo de potenciais evocados auditivos de tronco cerebral (BERA)e a ressonância nuclear magnética (RNM) (3). Na literatura existem autores que optam pela realização inicial do BERA, para só então, nos casos sugestivos de doença retrococlear, realizar RNM. Há outra corrente que indica a RNM como exame de eleição, uma vez que há casos de falso-negativos na realização do BERA. Desta forma, a redução de custo proporcionada por uma triagem inicial com o BERA não compensaria o diagnóstico tardio destas afecções e as possíveis complicações decorrentes.

\section{Relato de Caso}

JBS, 57 anos, sexo masculino, branco com história de hipoacusia e zumbido tipo apito bilateral há vários anos. Exame otorrinolaringológico apresentando otoscopia, rinoscopia anterior e orofaringe normais. Antecedentes de esquistossomose mansônica diagnosticada há 12 anos confirmada com parasitológico de fezes positivo em duas amostras para Schistosoma manısoni. Nega outras comorbidades. Realizada audiometrias tonal e vocal que evidenciaram perda neurossensorial assimétrica (Orelha direita: $500 \mathrm{~Hz}-40 \mathrm{~dB} ; 1000 \mathrm{~Hz}-35 \mathrm{~dB} ; 2000 \mathrm{~Hz}-40 \mathrm{~dB}$; $3000 \mathrm{~Hz}-40 \mathrm{~dB} ; 4000 \mathrm{~Hz}-35 \mathrm{~dB} ; 8000 \mathrm{~Hz}-50 \mathrm{db}$. Orelha esquerda: $500 \mathrm{~Hz}-65 \mathrm{~dB} ; 1000 \mathrm{~Hz}-65 \mathrm{~dB} ; 2000 \mathrm{~Hz}-70$ dB; 3000Hz - $75 \mathrm{~dB} ; 4000 \mathrm{~Hz}-75 \mathrm{~dB} ; 8000 \mathrm{~Hz}-85 \mathrm{~dB}$ ). Foi solicitada ressonância nuclear magnética que mostrou artéria basilar com ectasia e sinuosidade à esquerda, projetando-se para o ângulo ponto-cerebelar na fossa posterior, chegando a tocar na porção ventral da emergência do $7^{\circ} \mathrm{e}$ 8 o pares cranianos. Combase nos achados, foi estabelecido o diagnóstico de dolicoectasia de artéria basilar, sendo solicitada avaliação neurocirúrgica que afastou a possibilidade de tratamento cirúrgico considerando a topografia da lesão (alta morbimortalidade) e intensidade dos sintomas. A conduta escolhida foi acompanhamento do caso e prescrição de clonazepan na dosagem de $0,5 \mathrm{mg} /$ dia. Foi indicada ainda a utilização de aparelho de amplificação sonora individual na orelha esquerda.

\section{DISCUSSÃO}

Os distúrbios vasculares fazem parte do diagnóstico diferencial das perdas sensorioneurais assimétricas, dentre essas alterações encontra-se uma entidade rara conhecida como dolicomega da artéria basilar. Habitualmente a dolicomega é assintomática, porém quando há sintomas estes podem ser causados por compressão ou isquemia (4). Clinicamente podem ocorrer: perda neurossensorial (sendo raro como sintoma isolado), zumbido, cefaleia, hipoestesia facial, neuralgia trigeminal, vertigem, diplopia e paralisia facial entre outros (5-9). No caso estudado os sintomas foram de hipoacusia neurossensorial moderada em orelha esquerda acompanhada de zumbido bilateralmente sem outros sinais ou sintomas associados. O exame de imagem de escolha para seu diagnóstico é a ressonância nuclear magnética que pode demonstrar tortuosidade, estenose, trombose ou dolicoectasia (10). O paciente apresentou na RNM dolicomega da artéria basilar com sinuosidade. A mesma projetava-se para o ângulo pontocerebelar na fossa posterior estando bastante próxima da emergência dos nervos facial e vestíbulo-coclear. Há uma relação entre sintomatologia e achados nos exames de imagem. Quando se evidencia apenas tortuosidade sem dilatação torna-se mais provável o comprometimento de apenas um par craniano, no entanto, se há dilatação arterial importante pode haver o comprometimento múltiplo com déficits neurológicos graves (11). À época do diagnóstico, neste paciente não havia comprometimento de outros pares cranianos, além do vestíbulo-coclear, nem lesões neurológicas associadas. Podem ser encontrado eventualmente pacientes oligossintomáticos e com perdas auditivas leves apesar de terem lesões de dimensões acentuadas. Em 1986, Nishizaki et al estudaram 23 casos de dolicoectasia de artéria basilar em estudo retrospectivo num intervalo de 10 anos encontrando infarto pontino (30\%), insuficiência vértebro-basilar e de espasmo facial (17\%), ataque isquêmico transitório e de hemorragia cerebelar (4\%) (12). O trabalho conclui que a dolicoectasia da artéria basilar encontra-se associada com infarto isquêmico cerebral e que seria indicada, mesmo nos casos assintomáticos, terapia profilática contra acidente vascular cerebral isquêmico. Contudo já foi descrita também a compressão mecânica isolada por dolicomega de grandes dimensões causando a perda auditiva sem estar associada a infarto de tronco cerebral (13). Este achado parece ser semelhante ao que foi encontrado no paciente apresentado uma vez que não foram encontradas trombose nem aterosclerose no sistema vértebro-basilar que justificassem a perda auditiva descrita. É possível também que a perda auditiva esteja associada a comprometimento vestibular 
causando vertigem, transtornos visuais e oscilopsia associados a movimentos da cabeça e da marcha. Esses sintomas, entretanto não foram encontrados no paciente descrito. A terapia do dolicomega da artéria basilar será intervencionista ou conservadora dependendo dos a chados associados. A cirurgia pode ser perigosa pelo risco de causar lesão nos pequenos vasos que se originam na artéria basilar causando um acidente vascular. Já a terapia com antiagregantes plaquetários pode ser uma das estratégias adotadas para reduzir a possibilidade de trombose arterial (14). É fundamental uma abordagem multidisciplinar incluindo neurologista, neurocirurgião e otorrinolaringologista para adequada condução do caso uma vez que há uma grande variedade de apresentações clínicas desta doença e cujos tratamentos de escolha ainda permanecem controversos (15).

\section{COMENTÁRIOS FINAIS}

Nos casos de perda neurossensorial assimétrica acompanhada ou não de zumbido deverão fazer parte do diagnóstico diferencial as doenças de origem vascular, dentre elas o dolicomega da artéria basilar, cujo diagnóstico só será estabelecido com a investigação adequada.

\section{REFERÊNCIAS BIBLIOGRÁFICAS}

1. Benecke JE Jr, Hitselberger WE. Vertigo caused by basilar artery compression of the VIII nerve. Laryngoscope. 1988, 98:807-9.

2. Shin JH, Byun BJ, Kim DW, Choi DL. Neurenteric cyst in the cerebellopontine angle with xanthogranulomatous changes: serial MR findings with pathologic correlation. Am J Neuroradiol. 2002, 23:663-5.

3. Lemaire MC, Beutter P. Brainstem Auditory Evoked Responses in Patientes with Tinnitus. Audiology. 1995, 34:287-300.

4. Otterstedde CR, Tischendorf M, Reisser C. Megadolichobasilar artery as the etiology of sensorineural deafness in differential sudden deafness diagnosis] HNO. 1999, 47:494-6.
5. Lopez-DominguezJM, Casado-Chocan JL, Blanco-Ollero A, Rodriguez-Verdugo M, Robledo-Strauss A, Diaz-Espejo C. Bilateral hypoacusia and basilar dolichoectasia. Rev Neurol. 1996, 24(136):1538-40.

6. Cavallari FEM, Feres MCLC, Isac ML, Oliveira JAA. Tinnitus in a patient with dolichomegabasilar: a case report. Rev Bras Otorrinolaringol. 2004, 70(2):250-253.

7. Guiral H, RiscoJ, Ferrer F. Otoneurological manifestations of basilar dolicoectasia - a report of six cases: Acta Otorrinolaringol Esp. 1997, 48(5):337-40.

8. Jacobson DM, Corbett JJ. Downbeat nystagmus and dolichoectasia of the vertebrobasilar artery. J Neuroophthalmol. 2002, 22(2):150-1.

9. Buttner U, Ott M, Helmchen C, Yousry T. Bilateral loss of eighth nerve function as the only clinical sign of vertebrobasilar dolichoectasia. J Vestib Res. 1995, 5(1):4751.

10. Welsh LW, Welsh JJ, Lewin B. Basilar artery and vertigo. Ann Otol Rhinol Laryngol. 2000, 109(7):615-22.

11. Smoker WR, Corbett JJ, Gentry LR, Keyes WD, Price MJ, McKusker S. High-resolution computed tomography of the basilar artery: 2. Vertebrobasilar dolichoectasia: clinicalpathologic correlation and review. Am J Neuroradiol. 1986, 7(1):61-72.

12. Nishizaki T, Tamaki N, Takeda N, Shirakuni T, Kondoh T, Matsumoto S. Dolichoectatic basilar artery: a review of 23 cases. Stroke. 1986, 17:1277-81.

13. Liu CH, Lin SK, Chang YJ. Cochlear vertebral entrapment syndrome: a case report. Eur J Radiol. 2001, 40(2):147-50.

14. Campos CR, Doria-NettoHL, Souza-Filho AM, Silva Júnior HM. Dolicoectasia gigante e oligossintomática da artéria basilar descoberta após uma isquemia: relato de caso. Arq Neuro-Psiquiatr. 2007, 65(2A):345-7.

15. Meyerhoff WL, Mickey BE. Vascular decompression of the cochlear nerve. Laryngoscope. 1988, 98(6):602-4. 